\title{
Statistical Inference for Stochastic Differential Equations with Small Noises
}

\author{
Liang Shen ${ }^{1,2}$ and Qingsong $\mathrm{Xu}^{1}$ \\ ${ }^{1}$ School of Mathematics and Statistics, Central South University, Changsha, Hunan 410075, China \\ ${ }^{2}$ School of Science, Linyi University, Linyi, Shandong 276005, China \\ Correspondence should be addressed to Qingsong Xu; csuqingsongxu@126.com
}

Received 13 November 2013; Accepted 11 February 2014; Published 13 March 2014

Academic Editor: Zhi-Bo Huang

Copyright (c) 2014 L. Shen and Q. Xu. This is an open access article distributed under the Creative Commons Attribution License, which permits unrestricted use, distribution, and reproduction in any medium, provided the original work is properly cited.

\begin{abstract}
This paper proposes the least squares method to estimate the drift parameter for the stochastic differential equations driven by small noises, which is more general than pure jump $\alpha$-stable noises. The asymptotic property of this least squares estimator is studied under some regularity conditions. The asymptotic distribution of the estimator is shown to be the convolution of a stable distribution and a normal distribution, which is completely different from the classical cases.
\end{abstract}

\section{Introduction}

Stochastic differential equations (SDEs) are being extensively used as a model to describe some phenomena which are subject to random influences; it has found many applications in biology [1], medicine [2], econometrics [3, 4], finance [5], geophysics [6], and oceanography [7]. Then, statistical inference for these differential equations was of great interest and became a challenging theoretical problem. For a more recent comprehensive discussion, we refer to $[8,9]$.

The asymptotic theory of parametric estimation for diffusion processes with small white noise based on continuous time observations is well developed and it has been studied by many authors (see, e.g., [10-14]). There have been many applications of small noise in mathematical finance; see, for example, [15-18].

In parametric inference, due to the impossibility of observing diffusions continuously throughout a time interval, it is more practical and interesting to consider asymptotic estimation for diffusion processes with small noise based on discrete observations. There are many approaches to drift estimation for discretely observed diffusions (see, e.g., [1923]). Long [24] has started the study on parameter estimation for a class of stochastic differential equations driven by small stable noise $\left\{Z_{t}, t \geq 0\right\}$. However, there has been no study on parametric inference for stochastic processes with small Lévy noises yet.
In this paper, we are interested in the study of parameter estimation for the following stochastic differential equations driven by more general Lévy noise $\left\{L_{t}, t \geq 0\right\}$ based on discrete observations. We will employ the least squares method to obtain an asymptotically consistent estimator.

Let $\left(\Omega, \mathscr{F},\{\mathscr{F}\}_{t \geq 0}, \mathbb{P}\right)$ be a basic complete filtered probability space satisfying the usual conditions; that is, the filtration is continuous on the right and $\mathscr{F}_{0}$ contains all $\mathbb{P}$-null sets. In this paper, we consider a class of stochastic differential equations as follows:

$$
\begin{gathered}
d X_{t}=\theta f\left(X_{t}\right) d t+\varepsilon g\left(X_{t_{-}}\right) d L_{t}, \quad t \in[0,1], \\
L_{t}=a B_{t}+b Z_{t}, \\
X(0)=x_{0},
\end{gathered}
$$

where $f: \mathbb{R} \rightarrow \mathbb{R}$ and $g: \mathbb{R} \rightarrow \mathbb{R}$ are known functions and $a, b$ are known constants. Let $\left\{B_{t}, t \geq 0\right\}$ be a standard Brownian motion and let $\left\{Z_{t}, t \geq 0\right\}$ be a standard $\alpha$-stable Lévy motion independent of $\left\{B_{t}, t \geq 0\right\}$, with $Z_{1} \sim S_{\alpha}(1, \beta, 0)$ for $\beta \in[0,1], 1<\alpha<2$.

Let $X=\left\{X_{t}, t \geq 0\right\}$ be a real-valued, stationary process satisfying the stochastic differential equation (1) and we assume that this process is observed at regularly spaced time points $\left\{t_{i}=i / n, i=1,2, \ldots, n\right\}$. Assume $X_{t}^{0}$ is the solution of 
the underlying ordinary differential equation (ODE) with the true value of the drift parameter $\theta_{0}$ :

$$
d X_{t}^{0}=\theta_{0} f\left(X_{t}^{0}\right) d t, \quad X_{0}^{0}=x_{0} .
$$

Then, we get

$$
X_{t_{i}}-X_{t_{i-1}}=\int_{t_{i-1}}^{t_{i}} \theta_{0} f\left(X_{s}\right) d s+\varepsilon \int_{t_{i-1}}^{t_{i}} g\left(X_{s_{-}}\right) d L_{s} .
$$

\section{Preliminaries}

In this paper, we denote $C$ as a generic constant whose value may vary from place to place.

The following regularity conditions are assumed to hold:

$\left(\mathscr{A}_{1}\right)$ The functions $f(x)$ and $g(x)$ satisfy the Lipschitz conditions; that is, there exists a constant $L>0$ such that

$$
|f(x)-f(y)|+|g(x)-g(y)| \leq L|x-y|, \quad x, y \in \mathbb{R} .
$$

$\left(\mathscr{A}_{2}\right)$ There exist constants $M>0$ and $r \geq 0$ satisfying the growth condition

$$
g^{-2}(x) \leq M\left(1+|x|^{r}\right), \quad x \in \mathbb{R} .
$$

$\left(\mathscr{A}_{3}\right)$ There exists a positive constant $N>0$ such that $0<|g(x)| \leq N<\infty$.

$$
\begin{aligned}
& \left(\mathscr{A}_{4}\right) \text { For } C_{r}=2^{r-1} \vee 1, r>0, \\
& \quad\left|X_{t_{i-1}}\right|^{r} \leq C_{r}\left(\left|X_{t_{i-1}}^{0}\right|^{r}+\left|X_{t_{i-1}}-X_{t_{i-1}}^{0}\right|^{r}\right) .
\end{aligned}
$$

The LSE of $\widehat{\theta}_{n, \varepsilon}$ is defined as

$$
\widehat{\theta}_{n, \varepsilon}:=\underset{\theta}{\arg \min } \rho_{n, \varepsilon}(\theta)
$$

where the contrast function

$$
\rho_{n, \mathcal{\varepsilon}}(\theta)=\sum_{i=1}^{n}\left|\frac{X_{t_{i}}-X_{t_{i-1}}-\theta f\left(X_{t_{i-1}}\right) \Delta t_{i-1}}{\varepsilon g\left(X_{t_{i-1}}\right)}\right|^{2} .
$$

Then the $\widehat{\theta}_{n, \varepsilon}$ can be represented explicitly as follows:

$$
\widehat{\theta}_{n, \varepsilon}=\frac{\sum_{i=1}^{n} g^{-2}\left(X_{t_{i-1}}\right) f\left(X_{t_{i-1}}\right)\left(X_{t_{i}}-X_{t_{i-1}}\right)}{n^{-1} \sum_{i=1}^{n} g^{-2}\left(X_{t_{i-1}}\right) f^{2}\left(X_{t_{i-1}}\right)} .
$$

Based on (3) and (9), there is a special decomposition for $\widehat{\theta}_{n, \varepsilon}$ $\widehat{\theta}_{n, \varepsilon}$

$$
\begin{aligned}
= & \frac{\theta_{0} \sum_{i=1}^{n} g^{-2}\left(X_{t_{i-1}}\right) f\left(X_{t_{i-1}}\right) \int_{t_{i-1}}^{t_{i}} f\left(X_{s}\right) d s}{n^{-1} \sum_{i=1}^{n} g^{-2}\left(X_{t_{i-1}}\right) f^{2}\left(X_{t_{i-1}}\right)} \\
& +\frac{\varepsilon \sum_{i=1}^{n} g^{-2}\left(X_{t_{i-1}}\right) f\left(X_{t_{i-1}}\right) \int_{t_{i-1}}^{t_{i}} g\left(X_{s_{-}}\right) d L_{s}}{n^{-1} \sum_{i=1}^{n} g^{-2}\left(X_{t_{i-1}}\right) f^{2}\left(X_{t_{i-1}}\right)} \\
= & \theta_{0}+\frac{\theta_{0} \sum_{i=1}^{n} g^{-2}\left(X_{t_{i-1}}\right) f\left(X_{t_{i-1}}\right) \int_{t_{i-1}}^{t_{i}}\left(f\left(X_{s}\right)-f\left(X_{t_{i-1}}\right)\right) d s}{n^{-1} \sum_{i=1}^{n} g^{-2}\left(X_{t_{i-1}}\right) f^{2}\left(X_{t_{i-1}}\right)} \\
& +\frac{\varepsilon \sum_{i=1}^{n} g^{-2}\left(X_{t_{i-1}}\right) f\left(X_{t_{i-1}}\right) \int_{t_{i-1}}^{t_{i}} g\left(X_{s_{-}}\right) d L_{s}}{n^{-1} \sum_{i=1}^{n} g^{-2}\left(X_{t_{i-1}}\right) f^{2}\left(X_{t_{i-1}}\right)} \\
= & \theta_{0}+\frac{\theta_{0} \sum_{i=1}^{n} g^{-2}\left(X_{t_{i-1}}\right) f\left(X_{t_{i-1}}\right) \int_{t_{i-1}}^{t_{i}}\left(f\left(X_{s}\right)-f\left(X_{t_{i-1}}\right)\right) d s}{n^{-1} \sum_{i=1}^{n} g^{-2}\left(X_{t_{i-1}}\right) f^{2}\left(X_{t_{i-1}}\right)} \\
& +\frac{b \varepsilon \sum_{i=1}^{n} g^{-2}\left(X_{t_{i-1}}\right) f\left(X_{t_{i-1}}\right) \int_{t_{i-1}}^{t_{i}} g\left(X_{s_{-}}\right) d Z_{s}}{n^{-1} \sum_{i=1}^{n} g^{-2}\left(X_{t_{i-1}}\right) f^{2}\left(X_{t_{i-1}}\right)} \\
& +\frac{a \varepsilon \sum_{i=1}^{n} g^{-2}\left(X_{t_{i-1}}\right) f\left(X_{t_{i-1}}\right) \int_{t_{i-1}}^{t_{i}} g\left(X_{s_{-}}\right) d B_{s}}{n^{-1} \sum_{i=1}^{n} g^{-2}\left(X_{t_{i-1}}\right) f^{2}\left(X_{t_{i-1}}\right)} \\
= & \theta_{0}+\frac{\Phi_{2}(n, \varepsilon)}{\Phi_{1}(n, \varepsilon)}+\frac{\Phi_{3}(n, \varepsilon)}{\Phi_{1}(n, \varepsilon)}+\frac{\Phi_{4}(n, \varepsilon)}{\Phi_{1}(n, \varepsilon)} .
\end{aligned}
$$

Now we give an explicit expression for $\varepsilon^{-1}\left(\widehat{\theta}_{n, \varepsilon}-\theta_{0}\right)$. By using (10), we have

$$
\begin{aligned}
\mathcal{E}^{-1}\left(\widehat{\theta}_{n, \varepsilon}-\theta_{0}\right) & =\frac{\varepsilon^{-1} \Phi_{2}(n, \varepsilon)}{\Phi_{1}(n, \varepsilon)}+\frac{\varepsilon^{-1} \Phi_{3}(n, \varepsilon)}{\Phi_{1}(n, \varepsilon)}+\frac{\varepsilon^{-1} \Phi_{4}(n, \varepsilon)}{\Phi_{1}(n, \varepsilon)} \\
& :=\frac{\Psi_{2}(n, \varepsilon)}{\Phi_{1}(n, \varepsilon)}+\frac{\Psi_{3}(n, \varepsilon)}{\Phi_{1}(n, \varepsilon)}+\frac{\Psi_{4}(n, \varepsilon)}{\Phi_{1}(n, \varepsilon)} .
\end{aligned}
$$

One of the important tools we will employ is the underlying lemma (see (3.5) in the Lemma 3.2 of [24]).

Lemma 1. Under conditions $\left(\mathscr{A}_{1}\right)-\left(\mathscr{A}_{2}\right)$, one has

$$
\begin{gathered}
\left|X_{t}-X_{t}^{0}\right| \leq \varepsilon e^{L\left|\theta_{0}\right| t}\left|\int_{0}^{t} g\left(X_{s_{-}}\right) d Z_{s}\right|, \\
\sup _{0 \leq t \leq 1}\left|X_{t}-X_{t}^{0}\right| \longrightarrow{ }_{P} 0, \quad \text { as } \varepsilon \longrightarrow 0 .
\end{gathered}
$$




\section{Asymptotic Property of the Least Squares Estimator}

Theorem 2. Under the conditions $\left(\mathscr{A}_{1}\right)-\left(\mathscr{A}_{4}\right)$, as $n \rightarrow$ $\infty, \varepsilon \rightarrow 0, n \varepsilon \rightarrow \infty$, and $n \varepsilon^{\alpha /(\alpha-1)} \rightarrow \infty$, one has

$$
\begin{gathered}
\mathcal{E}^{-1}\left(\widehat{\theta}_{n, \varepsilon}-\theta_{0}\right) \\
\Longrightarrow a \frac{\left(\int_{0}^{1} g^{-2}\left(X_{s}^{0}\right) f^{2}\left(X_{s}^{0}\right) d s\right)^{1 / 2}}{\int_{0}^{1} g^{-2}\left(X_{s}^{0}\right) f^{2}\left(X_{s}^{0}\right) d s} N \\
+b\left(\left(\left(\int_{0}^{1}\left|g\left(X_{s}^{0}\right)\right|^{-2 \alpha}\left(f\left(X_{s}^{0}\right) g\left(X_{s}^{0}\right)\right)_{+}^{\alpha} d s\right)^{1 / \alpha} U_{1}\right.\right. \\
\left.-\left(\int_{0}^{1}\left|g\left(X_{s}^{0}\right)\right|^{-2 \alpha}\left(f\left(X_{s}^{0}\right) g\left(X_{s}^{0}\right)\right)_{-}^{\alpha} d s\right)^{1 / \alpha} U_{2}\right) \\
\left.\times\left(\int_{0}^{1} g^{-2}\left(X_{s}^{0}\right) f^{2}\left(X_{s}^{0}\right) d s\right)^{-1}\right),
\end{gathered}
$$

where $U_{1}$ and $U_{2}$ are independent random variables with $\alpha$ stable distribution $S_{\alpha}(1, \beta, 0)$ and $N$ is an independent random variable with standard normal distribution.

The theorem will be proved by establishing several propositions. We will consider the asymptotic behaviors of $\Phi_{1}(n, \varepsilon)$, $\Psi_{i}(n, \varepsilon), i=2,3,4$, respectively.

Proposition 3. Under conditions $\left(\mathscr{A}_{1}\right)-\left(\mathscr{A}_{4}\right)$, and $n \rightarrow \infty$, $\varepsilon \rightarrow 0$, one has

$$
\Phi_{1}(n, \varepsilon) \longrightarrow_{P} \int_{0}^{1} g^{-2}\left(X_{s}^{0}\right) f^{2}\left(X_{s}^{0}\right) d s
$$

Proof. Under conditions $\left(\mathscr{A}_{1}\right)-\left(\mathscr{A}_{3}\right)$, Proposition 3 can be proved by using condition $\left(\mathscr{A}_{4}\right)$ (see the proof of Proposition 3.3 in [24]).

Proposition 4. Under conditions $\left(\mathscr{A}_{1}\right)-\left(\mathscr{A}_{4}\right)$, as $n \rightarrow \infty$, $\varepsilon \rightarrow 0$ and $n \varepsilon \rightarrow \infty$, one has

$$
\Psi_{2}(n, \varepsilon) \longrightarrow{ }_{P} 0
$$

Proof. For $t_{i-1} \leq t \leq t_{i}, i=1,2, \ldots, n$,

$$
X_{t}=X_{t_{i-1}}+\int_{t_{i-1}}^{t} \theta_{0} f\left(X_{s}\right) d s+\varepsilon \int_{t_{i-1}}^{t} g\left(X_{s_{-}}\right) d L_{s}
$$

It follows that

$$
\begin{aligned}
& \left|X_{t}-X_{t_{i-1}}\right| \\
& \leq \int_{t_{i-1}}^{t}\left|\theta_{0}\right|\left(\left|f\left(X_{s}\right)-f\left(X_{t_{i-1}}\right)\right|+\left|f\left(X_{t_{i-1}}\right)\right|\right) d s \\
& \quad+\varepsilon\left|\int_{t_{i-1}}^{t} g\left(X_{s_{-}}\right) d L_{s}\right| \\
& \leq\left|\theta_{0}\right| M \int_{t_{i-1}}^{t}\left|f\left(X_{s}\right)-f\left(X_{t_{i-1}}\right)\right|+n^{-1}\left|\theta_{0}\right|\left|f\left(X_{t_{i-1}}\right)\right| \\
& \quad+a \varepsilon \sup _{t_{i-1} \leq t \leq t_{i}}\left|\int_{t_{i-1}}^{t} g\left(X_{s_{-}}\right) d B_{s}\right| \\
& \quad+b \varepsilon \sup _{t_{i-1} \leq t \leq t_{i}}\left|\int_{t_{i-1}}^{t} g\left(X_{s_{-}}\right) d Z_{s}\right| .
\end{aligned}
$$

Using Gronwall inequality, we get

$$
\begin{aligned}
& \left|X_{t}-X_{t_{i-1}}\right| \\
& \leq e^{\left|\theta_{0}\right| M\left(t-t_{i-1}\right)}\left[\frac{\left|\theta_{0}\right|\left|f\left(X_{t_{i-1}}\right)\right|}{n}\right. \\
& \quad+a \varepsilon \sup _{t_{i-1} \leq t \leq t_{i}}\left|\int_{t_{i-1}}^{t} g\left(X_{s_{-}}\right) d B_{s}\right| \\
& \left.+b \varepsilon \sup _{t_{i-1} \leq t \leq t_{i}}\left|\int_{t_{i-1}}^{t} g\left(X_{s_{-}}\right) d Z_{s}\right|\right],
\end{aligned}
$$

which yields

$$
\begin{aligned}
\sup _{t_{i-1} \leq t \leq t_{i}} \mid X_{t}- & X_{t_{i-1}} \mid \\
\leq e^{\left|\theta_{0}\right| M / n}[ & {\left[\frac{\left|\theta_{0}\right|\left|f\left(X_{t_{i-1}}\right)\right|}{n}\right.} \\
& +a \varepsilon \sup _{t_{i-1} \leq t \leq t_{i}}\left|\int_{t_{i-1}}^{t} g\left(X_{s_{-}}\right) d B_{s}\right| \\
& \left.+b \varepsilon \sup _{t_{i-1} \leq t \leq t_{i}}\left|\int_{t_{i-1}}^{t} g\left(X_{s_{-}}\right) d Z_{s}\right|\right],
\end{aligned}
$$

thus, under conditions $\left(\mathscr{A}_{1}\right)$ and $\left(\mathscr{A}_{3}\right)$,

$$
\begin{aligned}
& \left|\Phi_{2}(n, \varepsilon)\right| \\
& \leq\left|\theta_{0}\right| \sum_{i=1}^{n} M\left(1+\left|X_{t_{i-1}}\right|^{r}\right)\left|f\left(X_{t_{i-1}}\right)\right| \\
& \quad \times\left|\int_{t_{i-1}}^{t}\left(f\left(X_{s}\right)-f\left(X_{t_{i-1}}\right)\right) d s\right| \\
& \leq \frac{M K\left|\theta_{0}\right|}{n} \sum_{i=1}^{n}\left(1+\left|X_{t_{i-1}}\right|^{r}\right)\left|f\left(X_{t_{i-1}}\right)\right| \\
& \quad \times \sup _{t_{i-1} \leq t \leq t_{i}}\left|X_{t}-X_{t_{i-1}}\right|
\end{aligned}
$$




$$
\begin{aligned}
\leq & \frac{M K\left|\theta_{0}\right|^{2} e^{\left|\theta_{0}\right| M / n}}{n^{2}} \sum_{i=1}^{n}\left(1+\left|X_{t_{i-1}}\right|^{r}\right)\left|f\left(X_{t_{i-1}}\right)\right|^{2} \\
& +\frac{M K\left|\theta_{0}\right|^{2} e^{\left(\left|\theta_{0}\right| M\right) / n}}{n} b \varepsilon \sum_{i=1}^{n}\left(1+\left|X_{t_{i-1}}\right|^{r}\right)\left|f\left(X_{t_{i-1}}\right)\right| \\
& \times \sup _{t_{i-1} \leq t \leq t_{i}}\left|\int_{t_{i-1}}^{t} g\left(X_{s_{-}}\right) d Z_{s}\right| \\
& +\frac{M K\left|\theta_{0}\right|^{2} e^{\left|\theta_{0}\right| M / n}}{n} a \varepsilon \sum_{i=1}^{n}\left(1+\left|X_{t_{i-1}}\right|^{r}\right)\left|f\left(X_{t_{i-1}}\right)\right| \\
& \times \sup _{t_{i-1} \leq t \leq t_{i}}\left|\int_{t_{i-1}}^{t} g\left(X_{s_{-}}\right) d B_{s}\right| \\
:= & \Phi_{2,1}(n, \varepsilon)+\Phi_{2,2}(n, \varepsilon)+\Phi_{2,3}(n, \varepsilon) .
\end{aligned}
$$

Then,

$$
\begin{aligned}
\left|\Psi_{2}(n, \varepsilon)\right| \leq & \varepsilon^{-1} \Phi_{2,1}(n, \varepsilon)+\varepsilon^{-1} \Phi_{2,2}(n, \varepsilon) \\
& +\varepsilon^{-1} \Phi_{2,3}(n, \varepsilon) \\
:= & \Psi_{2,1}(n, \varepsilon)+\Psi_{2,2}(n, \varepsilon)+\Psi_{2,3}(n, \varepsilon) .
\end{aligned}
$$

Using (13) in Lemma 1 , conditions $\left(\mathscr{A}_{1}\right)$ and $\left(\mathscr{A}_{4}\right)$, we get $\Psi_{2,1}(n, \varepsilon) \rightarrow{ }_{P} 0$ as $n \rightarrow \infty, \varepsilon \rightarrow 0$ and $n \varepsilon \rightarrow \infty$ (see (3.26) in [24]). By using the same techniques, under condition $\left(\mathscr{A}_{2}\right)$, we can prove that $\Psi_{2, j}(n, \varepsilon) \rightarrow{ }_{p} 0, j=2,3$, as $n \rightarrow \infty, \varepsilon \rightarrow 0$, respectively.

Proposition 5. Under conditions $\left(\mathscr{A}_{1}\right)-\left(\mathscr{A}_{4}\right)$, as $n \rightarrow \infty$, $\varepsilon \rightarrow 0$ and $n \varepsilon^{\alpha /(\alpha-1)} \rightarrow \infty$, one has

$$
\begin{aligned}
& \Psi_{3}(n, \varepsilon) \\
& \quad \Longrightarrow b\left(\int_{0}^{1}\left|g\left(X_{s}^{0}\right)\right|^{-2 \alpha}\left(f\left(X_{s}^{0}\right) g\left(X_{s}^{0}\right)\right)_{+}^{\alpha} d s\right)^{1 / \alpha} U_{1} \\
& \quad-b\left(\int_{0}^{1}\left|g\left(X_{s}^{0}\right)\right|^{-2 \alpha}\left(f\left(X_{s}^{0}\right) g\left(X_{s}^{0}\right)\right)_{-}^{\alpha} d s\right)^{1 / \alpha} U_{2} .
\end{aligned}
$$

Proof. Under conditions $\left(\mathscr{A}_{1}\right)-\left(\mathscr{A}_{3}\right)$, Proposition 5 can be proved by using condition $\left(\mathscr{A}_{4}\right)$ (see the proof of Proposition 4.4 in [24]).

Proposition 6. Under conditions $\left(\mathscr{A}_{1}\right)-\left(\mathscr{A}_{4}\right)$, as $n \rightarrow \infty$, $\varepsilon \rightarrow 0$, one has

$$
\Psi_{4}(n, \varepsilon) \Longrightarrow a\left(\int_{0}^{1}\left|g^{-2}\left(X_{s}^{0}\right)\right| f^{2}\left(X_{s}^{0}\right) d s\right)^{1 / 2} N
$$

Proof. Note that

$$
\begin{aligned}
& \Psi_{4}(n, \varepsilon)=a \sum_{i=1}^{n} g^{-2}\left(X_{t_{i-1}}\right) f\left(X_{t_{i-1}}\right) \int_{t_{i-1}}^{t_{i}} g\left(X_{s_{-}}\right) d B_{s} \\
& =a \sum_{i=1}^{n} g^{-2}\left(X_{t_{i-1}}^{0}\right) f\left(X_{t_{i-1}}^{0}\right) \int_{t_{i-1}}^{t_{i}} g\left(X_{s_{-}}^{0}\right) d B_{s} \\
& +a \sum_{i=1}^{n} g^{-2}\left(X_{t_{i-1}}^{0}\right) f\left(X_{t_{i-1}}^{0}\right) \\
& \times \int_{t_{i-1}}^{t_{i}}\left(g\left(X_{s_{-}}\right)-g\left(X_{s_{-}}^{0}\right)\right) d B_{s} \\
& +a \sum_{i=1}^{n} g^{-2}\left(X_{t_{i-1}}^{0}\right)\left(f\left(X_{t_{i-1}}\right)-f\left(X_{t_{i-1}}^{0}\right)\right) \\
& \times \int_{t_{i-1}}^{t_{i}} g\left(X_{s_{-}}\right) d B_{s} \\
& +a \sum_{i=1}^{n}\left(g^{-2}\left(X_{t_{i-1}}\right)-g^{-2}\left(X_{t_{i-1}}^{0}\right)\right) f\left(X_{t_{i-1}}^{0}\right) \\
& \times \int_{t_{i-1}}^{t_{i}} g\left(X_{s_{-}}\right) d B_{s} \\
& +a \sum_{i=1}^{n}\left(g^{-2}\left(X_{t_{i-1}}\right)-g^{-2}\left(X_{t_{i-1}}^{0}\right)\right) \\
& \times\left(f\left(X_{t_{i-1}}\right)-f\left(X_{t_{i-1}}^{0}\right)\right) \\
& \times \int_{t_{i-1}}^{t_{i}} g\left(X_{s_{-}}\right) d B_{s} \\
& :=\sum_{j=1}^{5} \Psi_{4, j}(n, \varepsilon) \text {. }
\end{aligned}
$$

For $\Psi_{4,1}(n, \varepsilon)$, let $Y_{i}=\int_{t_{i-1}}^{t_{i}} g\left(X_{s_{-}}\right) d B_{s}, j=1, \ldots, n$. Then it is easy to see that $Y_{i} \sim N\left(0, \int_{t_{i-1}}^{t_{i}} g^{2}\left(X_{s_{-}}\right) d s\right)$ and $Y_{1}, \ldots, Y_{n}$ are independent normal random variables.

It follows that

$$
\begin{aligned}
& \Psi_{4,1}(n, \varepsilon) \\
& \quad=a \sum_{i=1}^{n} g^{-2}\left(X_{t_{i-1}}^{0}\right) f\left(X_{t_{i-1}}^{0}\right) Y_{i} \\
& \quad \sim N\left(0, a^{2} \sum_{i=1}^{n} g^{-4}\left(X_{t_{i-1}}^{0}\right) f^{2}\left(X_{t_{i-1}}^{0}\right) \int_{t_{i-1}}^{t_{i}} g^{2}\left(X_{s_{-}}\right) d s\right) \\
& \quad \Longrightarrow a\left(\int_{0}^{1} g^{-2}\left(X_{s}^{0}\right) f^{2}\left(X_{s}^{0}\right) d s\right)^{1 / 2} N
\end{aligned}
$$

as $n \rightarrow \infty, \varepsilon \rightarrow 0$. 
For $\Psi_{4,2}(n, \varepsilon)$, using Markov inequality and Ito's isometry property, for any given $\eta>0$,

$$
\begin{aligned}
\Psi_{4,2}( & n, \varepsilon) \\
\leq & \frac{1}{\eta} \mathbb{E}\left[a \sum_{i=1}^{n} g^{-2}\left(X_{t_{i-1}}^{0}\right)\left|f\left(X_{t_{i-1}}^{0}\right)\right|\right. \\
& \left.\times\left|\int_{t_{i-1}}^{t_{i}}\left(g\left(X_{s_{-}}\right)-g\left(X_{s_{-}}^{0}\right)\right) d B_{s}\right|\right] \\
\leq & \frac{a}{\eta} \sum_{i=1}^{n} g^{-2}\left(X_{t_{i-1}}^{0}\right)\left|f\left(X_{t_{i-1}}^{0}\right)\right| \\
& \times\left[\int_{t_{i-1}}^{t_{i}}\left(g\left(X_{s_{-}}\right)-g\left(X_{s_{-}}^{0}\right)\right)^{2} d s\right]^{1 / 2} \\
\leq & \frac{L a}{\eta} \sum_{i=1}^{n} g^{-2}\left(X_{t_{i-1}}^{0}\right)\left|f\left(X_{t_{i-1}}^{0}\right)\right| \\
& \times\left[\int_{t_{i-1}}^{t_{i}}\left(X_{s_{-}}-X_{s_{-}}^{0}\right)^{2} d s\right]^{1 / 2} \\
\leq & \frac{L a}{\eta} \sum_{i=1}^{n} g^{-2}\left(X_{t_{i-1}}^{0}\right)\left|f\left(X_{t_{i-1}}^{0}\right)\right| \\
& \times\left[\sup _{t_{i-1} \leq t \leq t_{i}}\left|X_{s_{-}}-X_{s_{-}}^{0}\right| n^{-1 / 2}\right] .
\end{aligned}
$$

By using (13), $\Psi_{4,2}(n, \varepsilon) \rightarrow 0$, as $n \rightarrow \infty, \varepsilon \rightarrow 0$.

Applying similar techniques to $\Psi_{4, j}(n, \varepsilon), j=3,4,5$, we get $\Psi_{4, j}(n, \varepsilon) \rightarrow 0, j=3,4,5$, as $n \rightarrow \infty, \varepsilon \rightarrow 0$.

Now we can prove Theorem 2.

Proof. By using Propositions 3, 4, 5, 6 and Slutsky's theorem, we can get the conclusion.

\section{Example}

We consider the following nonlinear SDE driven by general Lévy noises:

$$
d X_{t}=\theta X_{t} d t+\frac{\varepsilon}{1+X_{t_{-}}^{2}} d L_{t}, \quad t \in[0,1] ; \quad X_{0}=x_{0},
$$

where $f(x)=x, g(x)=1 /\left(1+x^{2}\right), x_{0}$ and $\varepsilon$ are known constants, and $\theta \neq 0$ is an unknown parameter.

For simplicity, let $x_{0}>0, \varepsilon=0$; we get the ODE:

$$
d X_{t}^{0}=\theta_{0} X_{t}^{0} d t, \quad t \in[0,1] ; \quad X_{0}^{0}=x_{0}
$$

and the solution

$$
X_{t}^{0}=x_{0} e^{\theta_{0} t}
$$

Then, the asymptotic distribution is

$$
\begin{aligned}
& a\left(\int_{0}^{1}\left(1+x_{0}^{2} e^{2 \theta_{0} s}\right)^{2} x_{0}^{2} e^{2 \theta_{0} s} d s\right)^{-1 / 2} N \\
& \quad+b \frac{\left(\int_{0}^{1}\left(1+x_{0}^{2} e^{2 \theta_{0} s}\right)^{\alpha} x_{0}^{\alpha} e^{\alpha \theta_{0} s} d s\right)^{1 / \alpha}}{\int_{0}^{1}\left(1+x_{0}^{2} e^{2 \theta_{0} s}\right)^{2} x_{0}^{2} e^{2 \theta_{0} s} d s} S_{\alpha}(1, \beta, 0)
\end{aligned}
$$

\section{Conflict of Interests}

The authors declare that there is no conflict of interests regarding the publication of this paper.

\section{References}

[1] R. I. Jennrich and P. B. Bright, "Fitting systems of linear differential equations using computer generated exact derivatives," Technometrics, vol. 18, no. 4, pp. 385-392, 1976.

[2] R. H. Jones, "Fitting multivariate models to unequally spaced data," in Time Series Analysis of Irregularly Observed Data, pp. 158-188, 1984.

[3] A. R. Bergstrom, Statistical Inference in Continuous Time Economic Models, vol. 99, North-Holland, Amsterdam, The Netherlands, 1976

[4] A. R. Bergstrom, "The history of continuous-time econometric models," Econometric Theory, vol. 4, no. 3, pp. 365-383, 1988.

[5] F. Black and M. Scholes, "The pricing of options and corporate liabilities," The Journal of Political Economy, vol. 81, pp. 637-654, 1973.

[6] M. Arató, Linear Stochastic Systems with Constant Coefficients, Springer, Berlin, Germany, 1982.

[7] R. J. Adler and P. Mèuller, Stochastic Modelling on Physical Oceanography, vol. 39, Springer, New York, NY, USA, 1996.

[8] B. P. Rao, B. L. P. Rao, I. Statisticien, B. L. P. Rao, B. L. P. Rao, and I. Statistician, Statistical Inference for di Usion Type Processes, Arnold, London, UK, 1999.

[9] Y. A. Kutoyants, Statistical Inference for Ergodic Diffusion Processes, Springer, London, UK, 2004.

[10] Yu. A. Kutoyants, Parameter Estimation for Stochastic Processes, vol. 6, Heldermann, Berlin, Germany, 1984.

[11] Yu. Kutoyants, Identification of Dynamical Systems with Small Noise, Kluwer Academic, Dordrecht, The Netherlands, 1994.

[12] N. Yoshida, "Asymptotic expansions of maximum likelihood estimators for small diffusions via the theory of MalliavinWatanabe," Probability Theory and Related Fields, vol. 92, no. 3, pp. 275-311, 1992.

[13] N. Yoshida, "Conditional expansions and their applications," Stochastic Processes and their Applications, vol. 107, no. 1, pp. 5381, 2003.

[14] M. Uchida and N. Yoshida, "Information criteria for small diffusions via the theory of Malliavin-Watanabe," Statistical Inference for Stochastic Processes, vol. 7, no. 1, pp. 35-67, 2004.

[15] N. Yoshida, "Asymptotic expansion of Bayes estimators for small diffusions," Probability Theory and Related Fields, vol. 95, no. 4, pp. 429-450, 1993.

[16] A. Takahashi, "An asymptotic expansion approach to pricing financial contingent claims," Asia-Pacific Financial Markets, vol. 6, no. 2, pp. 115-151, 1999. 
[17] N. Kunitomo and A. Takahashi, "The asymptotic expansion approach to the valuation of interest rate contingent claims," Mathematical Finance, vol. 11, no. 1, pp. 117-151, 2001.

[18] A. Takahashi and N. Yoshida, "An asymptotic expansion scheme for optimal investment problems," Statistical Inference for Stochastic Processes, vol. 7, no. 2, pp. 153-188, 2004.

[19] V. Genon-Catalot, "Maximum contrast estimation for diffusion processes from discrete observations," Statistics, vol. 21, no. 1, pp. 99-116, 1990.

[20] A. Gloter and M. Sørensen, "Estimation for stochastic differential equations with a small diffusion coefficient," Stochastic Processes and their Applications, vol. 119, no. 3, pp. 679-699, 2009.

[21] C. F. Laredo, "A sufficient condition for asymptotic sufficiency of incomplete observations of a diffusion process," The Annals of Statistics, vol. 18, no. 3, pp. 1158-1171, 1990.

[22] M. Uchida, "Approximate martingale estimating functions for stochastic differential equations with small noises," Stochastic Processes and their Applications, vol. 118, no. 9, pp. 1706-1721, 2008.

[23] M. Sørensen and M. Uchida, "Small-diffusion asymptotics for discretely sampled stochastic differential equations," Bernoulli, vol. 9, no. 6, pp. 1051-1069, 2003.

[24] H. Long, "Parameter estimation for a class of stochastic differential equations driven by small stable noises from discrete observations," Acta Mathematica Scientia B, vol. 30, no. 3, pp. 645-663, 2010. 


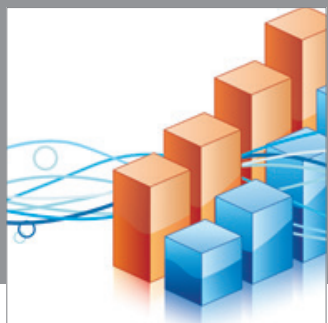

Advances in

Operations Research

mansans

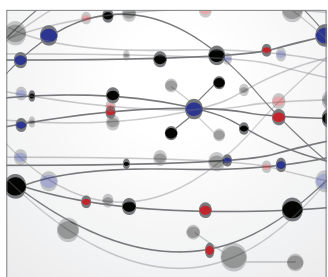

The Scientific World Journal
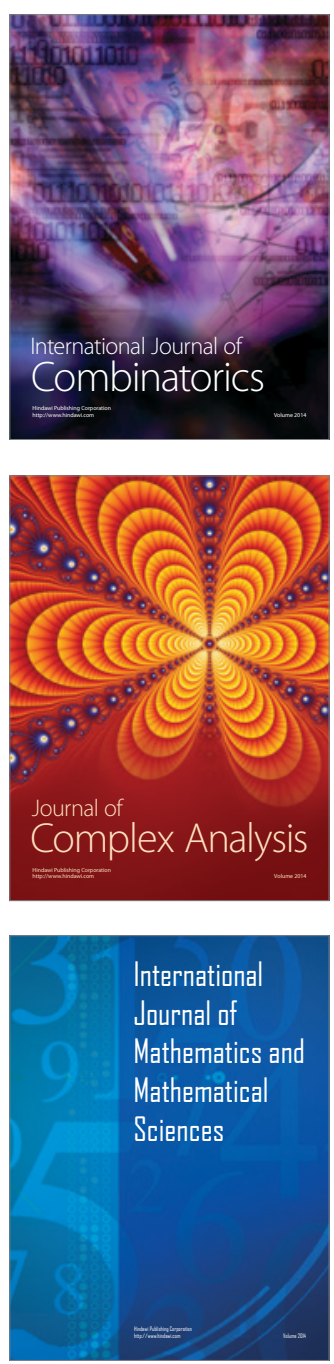
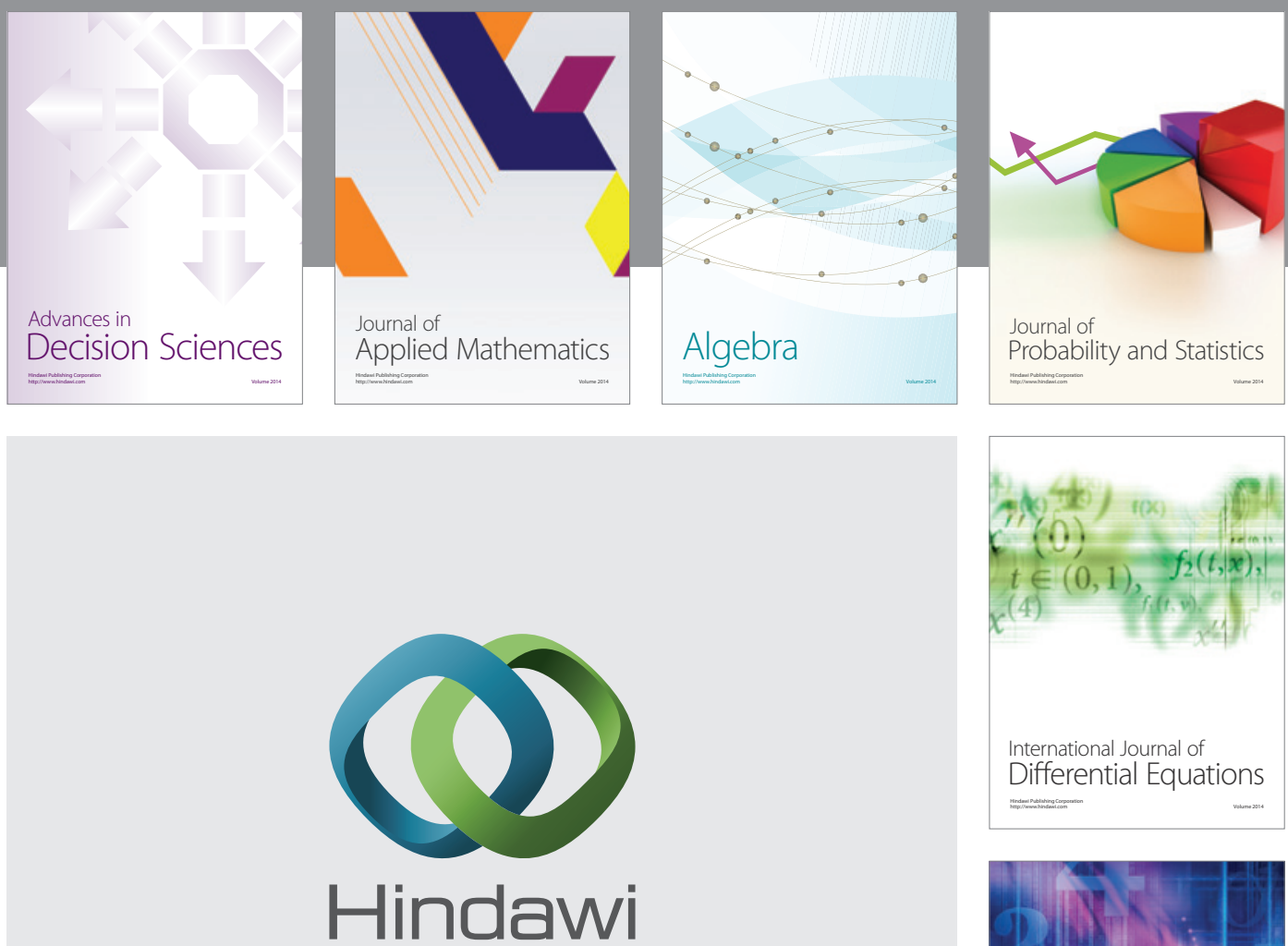

Submit your manuscripts at http://www.hindawi.com
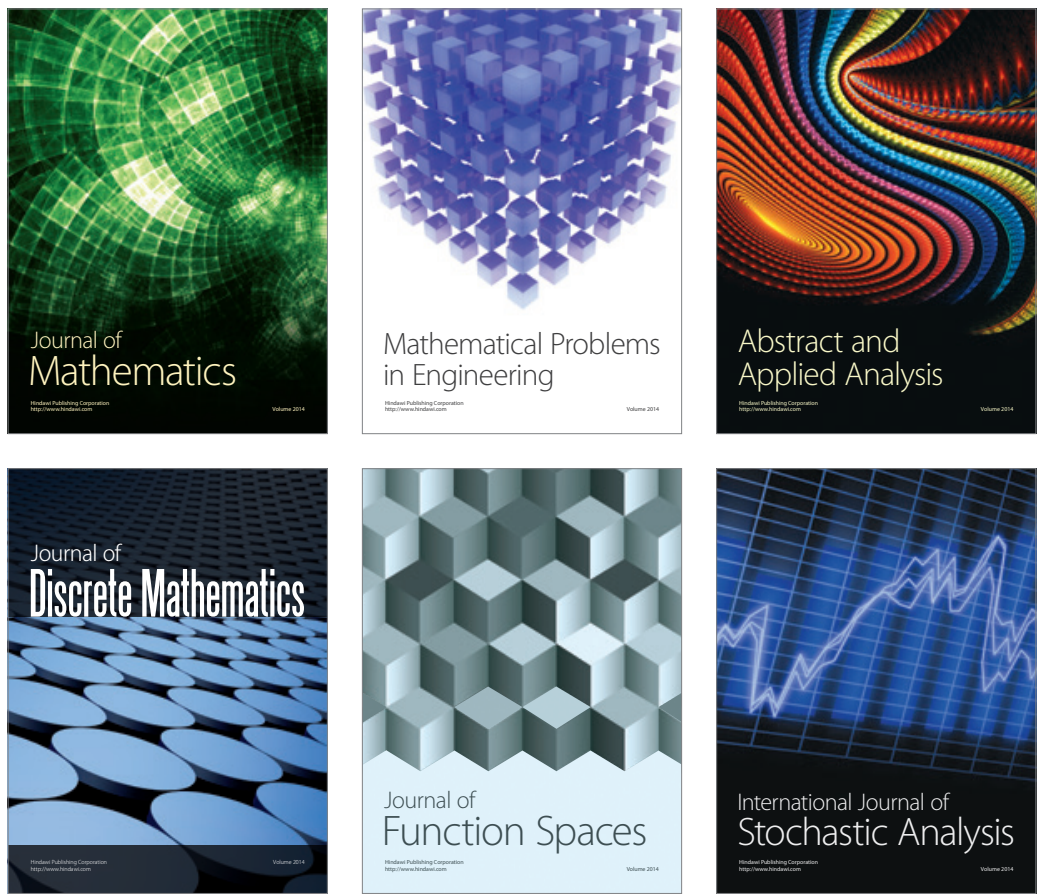

Journal of

Function Spaces

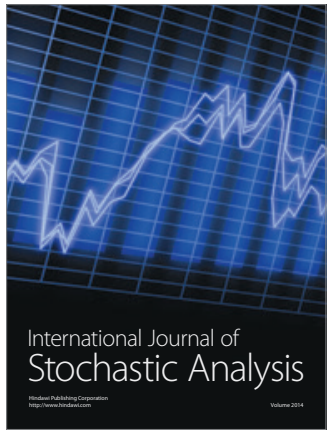

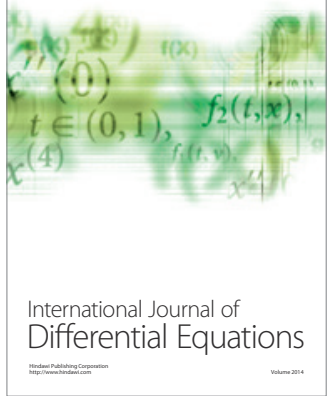
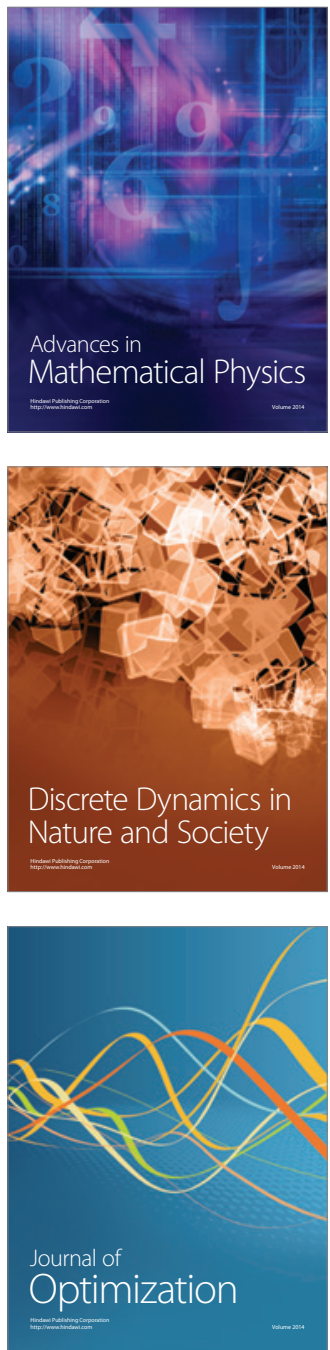